\title{
Modelling volatility of cryptocurrencies using Markov-Switching GARCH models
}

\author{
Guglielmo Maria Caporale ${ }^{\mathrm{a}, *}$, Timur Zekokh ${ }^{\mathrm{b}, 1}$ \\ ${ }^{\mathrm{a}}$ Brunel University London, United Kingdom \\ ${ }^{\mathrm{b}}$ Higher School of Economics, National Research University, Moscow, Russian Federation
}

\section{A R T I C L E I N F O}

\section{JEL classification:}

C22

G12

\section{Keywords:}

Cryptocurrencies

Volatility

Markov-switching

GARCH

\begin{abstract}
A B S T R A C T
This paper aims to select the best model or set of models for modelling volatility of the four most popular cryptocurrencies, i.e. Bitcoin, Ethereum, Ripple and Litecoin. More than 1000 GARCH models are fitted to the log returns of the exchange rates of each of these cryptocurrencies to estimate a one-step ahead prediction of Value-at-Risk (VaR) and Expected Shortfall (ES) on a rolling window basis. The best model or superior set of models is then chosen by backtesting VaR and ES as well as using a Model Confidence Set (MCS) procedure for their loss functions. The results imply that using standard GARCH models may yield incorrect VaR and ES predictions, and hence result in ineffective risk-management, portfolio optimisation, pricing of derivative securities etc. These could be improved by using instead the model specifications allowing for asymmetries and regime switching suggested by our analysis, from which both investors and regulators can benefit.
\end{abstract}

\section{Introduction}

Modelling volatility is crucial for risk management. Following the global financial crisis of 2008, the Basel III international regulatory framework for banks has imposed more stringent capital requirements, and enhanced risk management systems have been developed. Since then the international financial system has had to face a new challenge, namely the introduction of decentralised cryptocurrencies, the first being Bitcoin, which was created in 2009 (Nakamoto, 2009). Unlike traditional currencies, cryptocurrencies are based on cryptographic proof, which provides many advantages over traditional payment methods (such as credit cards) including high liquidity, lower transaction costs, and anonymity (these features are discussed by Fantazzini et al., 2016).

Interest in Bitcoin and other cryptocurrencies has risen considerably in recent years. Their market capitalisation increased from approximately 18 billion US dollars at the beginning of 2017 to nearly 600 billion at the end of that year ${ }^{2}$, and high returns have attracted new investors. In addition, two big exchanges, i.e. the Chicago Mercantile Exchange (CME) and the Chicago Board Options Exchange (CBOE), started to trade futures on Bitcoin. ${ }^{3}$ As a result of these developments, central banks have been facing the question of whether or not cryptocurrencies should be regulated, given the numerous technical and legal issues involved.

Further, cryptocurrencies are highly volatile and consequently it is important to estimate appropriate risk metrics, which can be used for calculating capital requirements, margins, hedging and pricing derivatives etc. It is well known that standard GARCH models

\footnotetext{
* Corresponding author at: Department of Economics and Finance, Brunel University, London, UB8 3PH, United Kingdom.

E-mail address: Guglielmo-Maria.Caporale@brunel.ac.uk (G.M. Caporale).

${ }^{1}$ The second-named author gratefully acknowledges financial support from the Russian Academic Excellence Project '5-100'.

${ }^{2}$ Source: https://coinmarketcap.com/

${ }^{3}$ Source: Thomson Reuters
} 
can produce biased results if the series display structural breaks (Bauwens et al. (2010, 2014); these are likely to occur in the case of cryptocurrencies. Specifically, Chu et al. (2017) argue that structural changes not accounted for (such as policy changes) might explain why the Integrated GARCH $(1,1)$ (IGARCH) model is found to be a good fit for numerous cryptocurrencies (Caporale et al. (2003)). Therefore a more suitable modelling approach should be used. Ardia et al. (2018a) suggest estimating in such cases MarkovSwitching GARCH (MSGARCH) models, whose parameters can change over time according to a discrete latent variable.

The aim of this paper is to find the best model or set of models for modelling volatility of the four most popular cryptocurrencies, i.e. Bitcoin, Ethereum, Ripple and Litecoin. More than $1000 \mathrm{GARCH}$ models are fitted to the log returns of the exchange rates of each of these cryptocurrencies to estimate a one-step ahead prediction of Value-at-Risk (VaR) and Expected Shortfall (ES) on a rolling window basis. The best model or superior set of models is then chosen by backtesting VaR and ES as well as using a Model Confidence Set (MCS) procedure for their loss functions.

The paper is organised as follows. Section 2 briefly discusses the relevant literature; Section 3 provides a description of the data; Section 4 outlines the methodology; Section 5 presents the empirical results; finally, Section 6 offers some concluding remarks.

\section{Literature review}

Generalized Autoregressive Conditional Heteroskedasticity (GARCH) models are the most commonly used in the literature for modelling volatility and estimating Value-at-Risk (VaR) and Expected Shortfall (ES). The original Autoregressive Conditional Heteroskedasticity (ARCH) specification was introduced by Engle (1982) and then extended by Bollerslev (1986), who put forward the GARCH framework. Additional specifications were then developed: the exponential GARCH (EGARCH) model of Nelson (1991), the threshold GARCH (TGARCH) model of Zakoian (1994), the Student's t-GARCH model of Bollerslev (1987), the GJRGARCH model of Glosten et al. (1993) and many others (see Bollerslev et al. (1992), 1994 and Engle (2004)). Hansen and Lunde (2005) showed that a GARCH $(1,1)$ specification outperforms more sophisticated models in the case of exchange rates, whilst models including a leverage effect were found to be more appropriate for IBM returns.

The first studies using a Markov-switching framework for modelling volatility estimated ARCH specifications (Cai (1994); Hamilton and Susmel (1994)) because of their computational tractability compared to GARCH ones; in particular, the evaluation of the likelihood function for Markov-switching GARCH model required integration over all $K^{N}$ paths (where $K$ is the number of regimes and $N$ is the number of observations), making such estimation extremely difficult. To solve this problem Dueker (1997); Gray (1996) and Klaassen (2002) used an ad hoc approximation method based on collapsing the past regime-specific conditional variances.

Recent studies have shown that structural breaks result in biased estimates of GARCH models and poor volatility forecasts (Bauwens et al. (2010, 2014). To deal with this issue Markov-switching GARCH models (MSGARCH) have been proposed, whose parameters can change over time according to a discrete latent (i.e., unobservable) variable. In particular, Ardia et al. (2018a) estimated a large set of MSGARCH specifications allowing for various functional forms as well as distributions of the errors. They compared two estimation techniques, namely Maximum Likelihood (ML) and Markov chain Monte Carlo (MCMC) procedures, and found that MSGARCH outperforms single regime models in forecasting VaR and ES for daily, weekly, and ten-day equity log-returns. Ardia et al. (2018a) followed Haas et al. (2004) in letting the GARCH process of each state evolve independently of those in the other states - this is also the method used in the current study.

Markov-switching GARCH models have been used in recent papers to analyse various type of assets: commodity prices (Alizadeh et al. (2008)), stock returns (Henry (2009)), exchange rate returns (Wilfling (2009); Bohl et al. (2011)), etc.

As for volatility modelling in the case of cryptocurrencies, many studies have relied on the GARCH framework. Glaser et al. (2014) estimated a standard GARCH $(1,1)$. Gronwald (2014) reported that an autoregressive jump-intensity GARCH model fits the Bitcoin data better than a standard GARCH. Dyhrberg (2016) estimated an asymmetric GARCH for Bitcoin arguing that it can be used for hedging. Bouoiyour and Selmi (2016) compared different specifications including EGARCH, Asymmetric Power ARCH (APARCH), weighted GARCH and component GARCH with multiple thresholds by using in-sample criteria such as the Akaike information criterion (AIC), the Bayesian information criterion (BIC) and the Hannan-Quinn information criterion (HQC); they concluded that, despite a noticeable decrease in its volatility, Bitcoin cannot yet be considered a mature currency. Katsiampa (2017) found that the AR-CGARCH model gives the best fit for Bitcoin, which means that accounting for both the short- and long-term components of the conditional variance is important. Chu et al. (2017) fitted 12 GARCH specifications with different distributions for the innovation process to seven cryptocurrencies; an IGARCH $(1,1)$ model with normal innovations was found to yield the smallest values of AIC, the corrected AIC (AICc), BIC, HQC and the Consistent AIC (CAIC) in the case of Bitcoin, Dash, Litecoin, Maidsafecoin and Monero; instead the GJRGARCH $(1,1)$ and GARCH $(1,1)$ model with normal innovations produced the smallest values for Dogecoin and Ripple respectively.

Ardia et al. (2018b) have instead tested the presence of regime changes in the GARCH volatility dynamics of Bitcoin using MSGARCH models. Specifically, they use daily Bitcoin mid-prices, which are then transformed into log-returns. Both in-sample and out-of-sample analysis are carried out, and Bitcoin log-returns are found to exhibit regime changes in their volatility dynamics, with MSGARCH models outperforming standard single-regime GARCH models. Our analysis below extends the study by Ardia et al. (2018b) by analysing three other cryptocurrencies (Litecoin, Ethereum, Ripple) in addition to Bitcoin, using ES and joint loss functions as well as the MCS procedure advocated by Hansen et al. (2011), and considering a much larger set of models.

\section{Data description}

The series analysed are the daily closing prices of Bitcoin, Ethereum, Ripple and Litecoin. The Bitcoin data were taken from the 
Coindesk Price Index and cover the period from 18 July 2010 to 30 April of 2018; for the other three series the data source is CoinMarketCap. ${ }^{4}$ The end date is the same for all series, whilst the start date differs: it is 7 August 2015 for Ethereum, 4 August 2013 for Ripple, and 28 April 2013 for Litecoin. Prices were transformed into log returns by taking first differences of their logarithm (see Fig. 1).

Summary statistics for the log returns are shown in Table 1.

As can be seen, they are negatively skewed in the case of Bitcoin and positively skewed in all other cases. All four cryptocurrencies exhibit leptokurtosis. The histograms of the log returns series are shown in Fig. 2.

\section{Methodology}

\subsection{GARCH models}

Let $y_{t} \in R$ be the percentage log-returns of the financial asset (exchange rate) of interest at time $t$. Following Ardia et al. (2018a), we adopt the following general Markov-Switching GARCH specification:

$$
y_{t} \mid\left(S_{t}=k, I_{t-1}\right)^{\sim} F\left(0, h_{k, t}, \theta_{k}\right),
$$

where $F\left(0, h_{k, t}, \theta_{k}\right)$ is a continuous distribution with zero mean, time-varying variance $h_{k, t}$, and additional shape parameters contained in the vector $\theta_{k}$. Following Ardia et al. (2018a), the integer-valued stochastic variable $S_{t}$, defined on the discrete space $\{1, \ldots$, $\mathrm{K}\}$, is assumed to evolve according to an unobserved first-order ergodic homogeneous Markov chain with transition probability matrix $\boldsymbol{P}=\left\{p_{i, j}\right\}_{i, j=1}^{k}$, with $p_{i, j}=P\left[S_{t}=j \mid S_{t-1}=i\right]$, with $I_{t-1}$ being the information set available at time $t-1$.

As in Haas et al. (2004), the conditional variance of $y_{t}$ is assumed to follow a GARCH process. This is not restricted to be the standard GARCH model:

$$
h_{k, t}=\omega\left(y_{t-1}, h_{k, t-1}, \theta_{k}\right) \text {, }
$$

where $\omega(\cdot)$ - is a $I_{t-1}$ - measurable function which defines the filter for the conditional variance and also ensures its positiveness. By contrast, a variety of GARCH specifications are considered, in particular:

SGARCH (Bollerslev (1986))

$$
\sigma_{t}^{2}=\omega+\sum_{i=1}^{p} \alpha_{i} \varepsilon_{t-i}^{2}+\sum_{j=1}^{q} \beta_{j} \sigma_{t-j}^{2}
$$

EGARCH (Nelson (1991))

$$
\ln \left(\sigma_{t}^{2}\right)=w+\sum_{i=1}^{p}\left\{\alpha_{i} *\left(\frac{\left|\varepsilon_{t-i}\right|}{\sigma_{t-i}}-E\left[\frac{\left|\varepsilon_{t-i}\right|}{\sigma_{t-i}}\right]\right)+\gamma_{i} \frac{\varepsilon_{t-i}}{\sigma_{t-i}}\right\}+\sum_{j}^{q} \ln \left(\sigma_{t-j}^{2}\right)
$$

GJRGARCH (Glosten et al. (1993))

$$
\sigma_{t}^{2}=\omega+\sum_{i=1}^{p}\left[\alpha_{i}+\gamma_{i} I_{\left(\varepsilon_{t-i}>0\right)}\right] \varepsilon_{t-i}^{2}+\sum_{1}^{q} \beta_{j} \sigma_{t-j}^{2}
$$

TGARCH (Zakoian (1994))

$$
\sigma_{t}^{2}=\omega+\sum_{i=1}^{p} \alpha_{i}\left[\left(1-\gamma_{i}\right) \varepsilon_{t-i}^{+}-\left(1+\gamma_{i}\right) \varepsilon^{-}\right]+\sum_{j=1}^{q} \beta_{j} \sigma_{t-j}^{2}
$$

As for distribution mixture models, suppose that:

$$
y_{t}^{\sim} D M\left(p_{1}, \ldots, p_{k} ; \mu_{1}, \ldots, \mu_{k} ; h_{1}, \ldots, h_{k}\right) \text {, }
$$

where DM is a mixture of densities with the following form:

$$
\vartheta(y)=\sum_{1}^{k} p_{i} f_{i}(y), \sum_{1}^{k} p_{i}=1, f_{i}(y)=f\left(y ; \mu_{i} ; h_{i}\right),
$$

where $\left[p_{1}, \ldots, p_{k}\right]$ is the positive mixing law and $f$ denotes the density function.

As an example of a distribution mixture model, consider the normal mixture models: according to Alexander and Lazar (2006), these can be seen as Markov switching GARCH models in a restricted form, where the transition probabilities are independent of the past state. It is assumed that $K$ variances follow some mixture of distributions. For example, the normal mixture standard GARCH (1, 1 ) will be defined as follows:

$$
\sigma_{i t}^{2}=\omega_{i}+\alpha_{i} \varepsilon_{t-1}^{2}+\beta_{i} \sigma_{i t-1}^{2}, \text { for } i=1, \ldots, K .
$$

\footnotetext{
${ }^{4}$ https://coinmarketcap.com/
} 

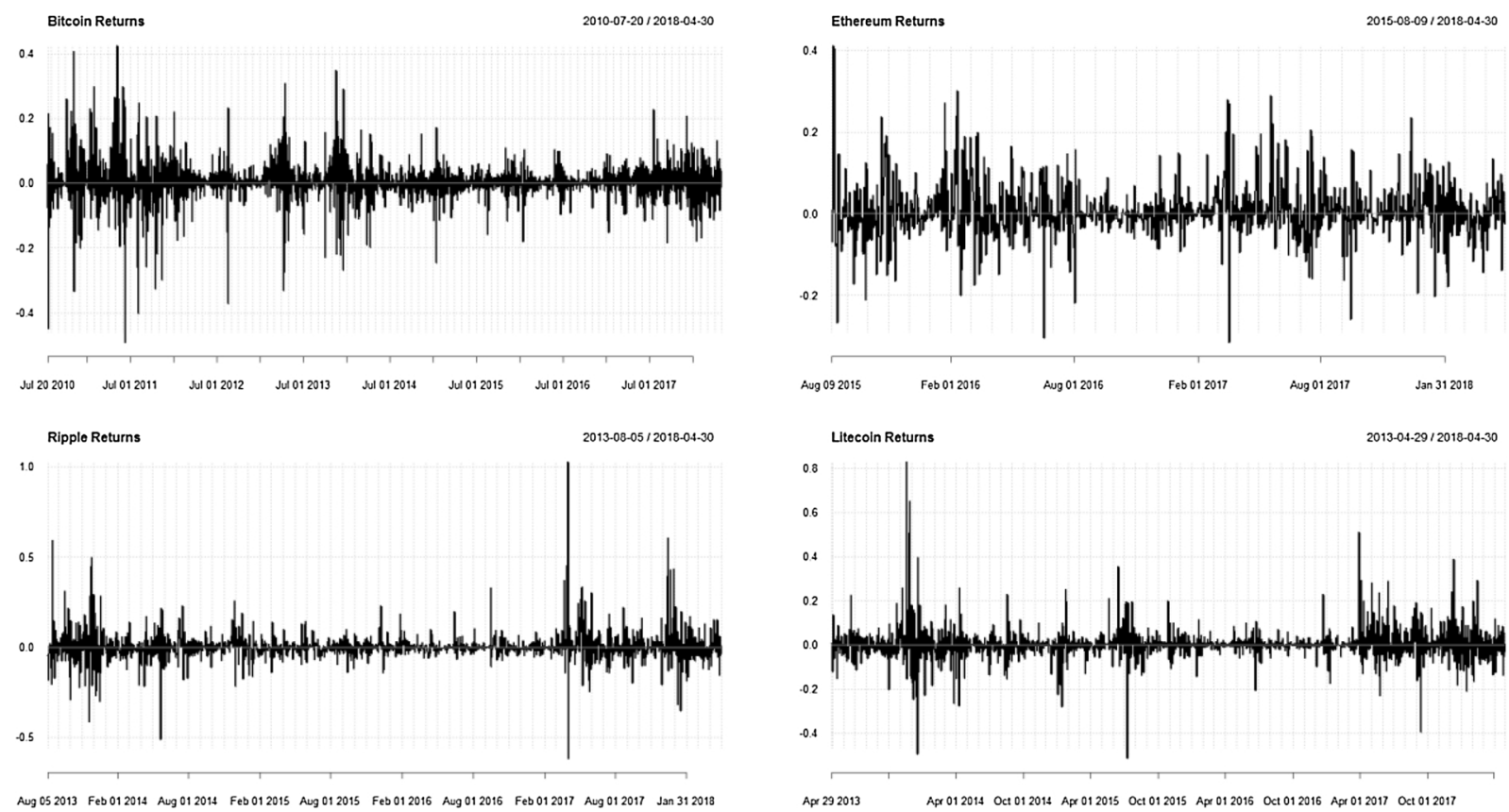

Fig. 1. Log returns.

Table 1

Descriptive statistics of log returns.

\begin{tabular}{lllll}
\hline Statistics & Bitcoin & Ethereum & Ripple \\
\hline Number of obs. & 2842 & 996 & 1730 & Litecoin \\
Minimum & -0.491 & -0.315 & -0.616 & -0.023 \\
Q1 & -0.013 & -0.027 & -0.003 & -0.514 \\
Median & 0.002 & 0 & 0.003 & 0.019 \\
Mean & 0.004 & 0.007 & 0.022 & 0.002 \\
Q3 & 0.023 & 0.036 & 1.027 & 0.018 \\
Maximum & 0.425 & 0.412 & 0.080 & 0.829 \\
Std Dev. & 0.058 & 0.072 & 1.998 & 0.069 \\
Skewness & -0.326 & 0.515 & 26.629 & 24.961 \\
Kurtosis & 11.811 & 4.347 & & 2 \\
\hline
\end{tabular}

According to the Cifter and Ozun (2007) the overall conditional variance will then be:

$$
\sigma_{t}^{2}=\sum_{i=1}^{K} p_{i} \sigma_{i t}^{2}+\sum_{i=1}^{K} p_{i} \mu_{i}^{2}
$$

\subsection{Value-at-risk backtesting}

Value-at-Risk at level $\alpha$ is defined as the maximum loss one could expect to incur with probability $\alpha$ over a specific period. Mathematically:

$$
\operatorname{VaR}_{\alpha}(X)=\inf \left\{x F_{X}(x) \geq \alpha\right\}=F_{X}^{-}(x),
$$

where $\operatorname{VaR}_{\alpha}(X)$ is $\operatorname{VaR}$ at level $\alpha$ of a random variable $X, F_{X}(x)$ is the cumulative distribution function of $X$. Basically, VaR is the $\alpha$ -quantile of the underlying distribution.

For carrying out VaR forecast tests, the first step is to define the "hit sequence" of VaR violations:

$$
\begin{aligned}
& I_{t+1}=1, \text { if } r_{t+1}<-V a R_{t+1}^{\alpha}, \\
& I_{t+1}=0, \text { if } r_{t+1}>-V a R_{t+1}^{\alpha},
\end{aligned}
$$

where $\operatorname{VaR}_{t+1}^{a}$ is the VaR prediction at time $t+1$ for risk level $\alpha$. Under the null hypothesis of correct specification the hit sequence should be an independent Bernoulli distributed variable. 

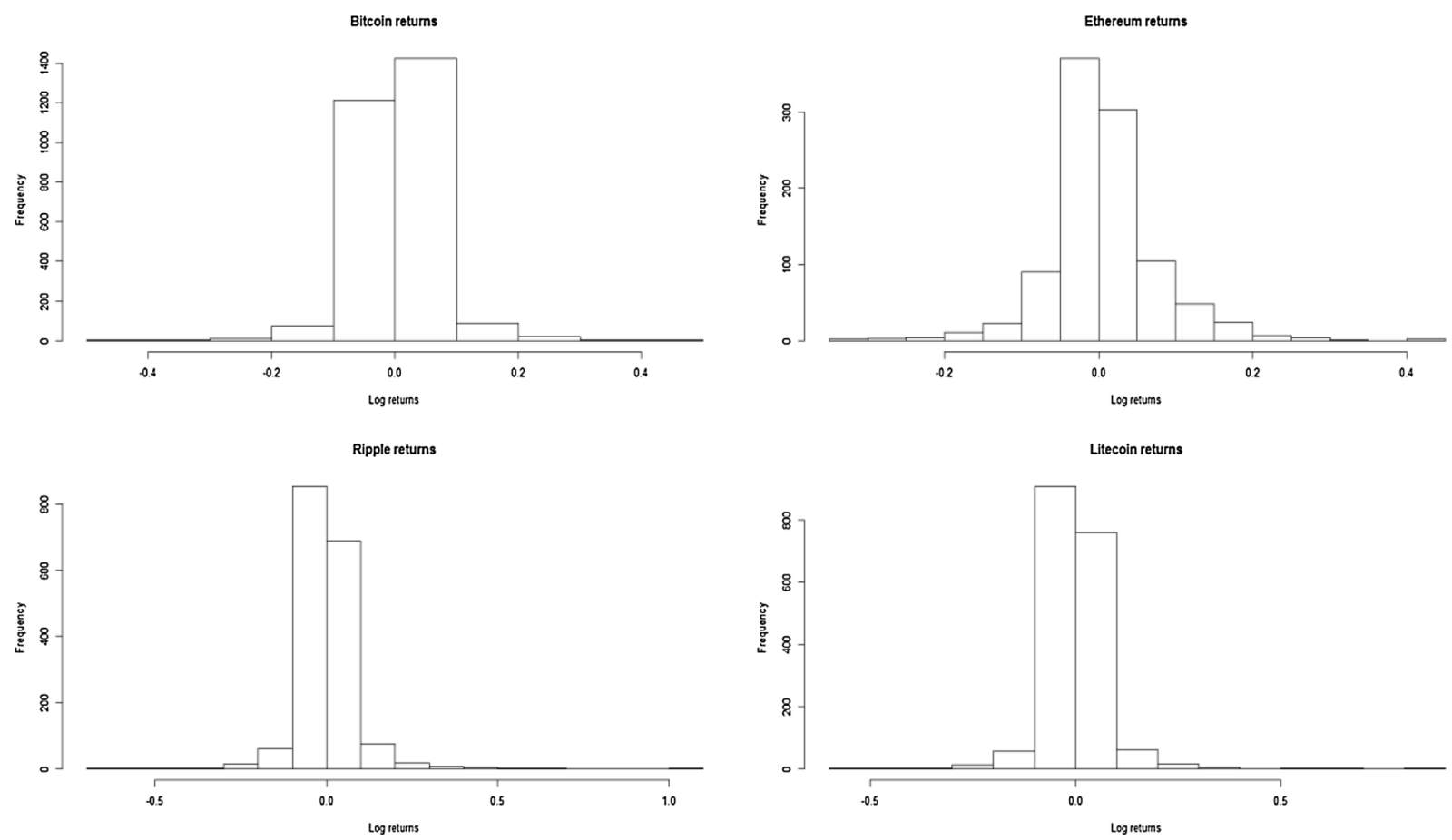

Fig. 2. Histograms of $\log$ returns.

$H_{0}: I_{t+1} \sim \operatorname{Bernoulli}(\alpha)$,

$$
f\left(I_{t+1}, p\right)=(1-p)^{1-I_{t+1}} p^{I_{t+1}}
$$

The unconditional coverage (UC) test of Kupiec (1995) uses the fraction of observed violations for a particular risk model $-\pi$ and compares it with $p$. For this purpose the likelihood Bernoulli function is needed:

$$
L(\pi)=\Pi(1-\pi)^{1-I_{t+1}} \pi^{I_{t+1}}=(1-\pi)^{T_{0}} \pi^{T_{1}},
$$

where $T_{0}, T_{1}$ are the number of $0 \mathrm{~s}$ and $1 \mathrm{~s}$ in the sample $\left(T=T_{0}+T_{1}\right)$. The maximum likelihood estimator is:

$$
\hat{\pi}=\frac{T_{1}}{T_{0}+T_{1}}
$$

The hypothesis of interest can be tested by means of the following likelihood ratio test:

$$
L R_{u c}=-2 \ln \left[\frac{L(\alpha)}{L(\hat{\pi})}\right] \chi_{1}^{2},
$$

Under the null hypothesis that the model is correct $L R_{u c}$ is asymptotically $\chi_{1}^{2}$.

However, this test focuses only on the number of exceptions. A situation can arise when the model passes the unconditional coverage test but all violations are concentrated. In order to reject a VaR with clustered violations a test of independence of the hit sequence is needed. Suppose that this exhibits time dependence and follows a first-order Markov sequence with the following transition probability matrix:

$$
\Pi_{1}=\left(\begin{array}{ll}
1-\pi_{01} & \pi_{01} \\
1-\pi_{11} & \pi_{11}
\end{array}\right),
$$

where $\pi_{01}$ is the probability of getting a violation tomorrow given no violation today.

Then the corresponding likelihood function is the following:

$$
L\left(\Pi_{1}\right)=\left(1-\pi_{01}\right)^{T_{00}} \pi_{01}^{T_{01}}\left(1-\pi_{11}\right)^{T_{10}} \pi_{11}^{T_{11}},
$$

where $T_{i j}$ is the number of observations with a $j$ following $i$.

If the hit sequence is independent over time, then $\pi_{01}=\pi_{11}=\pi$. The transition probability matrix will have the following form:

$$
\hat{\Pi}=\left(\begin{array}{ll}
1-\hat{\pi} & \hat{\pi} \\
1-\hat{\pi} & \hat{\pi}
\end{array}\right)
$$

Then, independence can be tested using a likelihood ratio test statistic defined as follows: 


$$
L R_{\text {ind }}=-2 \ln \left[\frac{L(\hat{\pi})}{L\left(\hat{\Pi}_{1}\right)}\right] \chi_{1}^{2} .
$$

It is important for VaR users to be able to test simultaneously whether the hit sequence is independent and the average number of violations is correct; the conditional coverage (CC) test developed by Christoffersen (1998) makes this possible. A sequence of VaR forecasts at risk level $\alpha$ has the correct conditional coverage if $\left\{I_{t}^{a} ; t=1, \ldots, H\right\}$ is an independent and identically distributed sequence of Bernoulli random variables with parameter $\alpha$. To test this hypothesis a joint test of independence of the series and the unconditional coverage of the VaR forecasts the likelihood ratio defined as follows is carried out:

$$
L R_{c c}=-2 \ln \left[\frac{L(\alpha)}{L\left(\hat{\Pi}_{1}\right)}\right] \chi_{2}^{2} .
$$

This is equivalent to testing the null $\pi_{01}=\pi_{11}=\alpha$. Note also that $L R_{c c}=L R_{u c}+L R_{\text {ind }}$.

An additional test is the dynamic quantile (DQ) one introduced by Manganelli and Engle (2004). It is based on a linear regression of the hit variable on a set of explanatory variables including a constant, the lagged values of the hit variable and any useful function of past information. Let us denote $\operatorname{Hit}_{t}(\alpha)=I_{t}(\alpha)-\alpha$. Under the correct model specification, the following moment conditions are satisfied: $E\left[H_{i t}(\alpha)\right]=0, E\left[H_{i t}(\alpha) I_{t-1}\right]=0, E\left[H_{i t}(\alpha) H_{t} t_{t}(\alpha)\right]=0$ for $t \neq t^{\prime}$. The linear regression is the following:

$$
H i t_{t}(\alpha)=\delta+\sum_{k=1}^{K} \beta_{k} H i t_{t-1}(\alpha)+\sum_{k=1}^{K} \gamma_{k} g\left[H i t_{t-k}(\alpha), H i t_{t-k-1}(\alpha), \ldots\right]+\varepsilon_{t},
$$

where $g($.$) is a function of past information. The null hypothesis of conditional efficiency is equivalent to testing whether the$ coefficients are jointly equal to zero:

$$
H_{0}: \delta=\beta_{1}=\ldots=\beta_{k}=\gamma_{1}=\ldots=\gamma_{k}=0, \forall k=1, \ldots, K
$$

However, these tests do not provide any insights into the magnitude of exceedance and therefore do not enable the researcher to make model comparisons.

A final test is due to González-Rivera et al. (2004) and McAleer and Da Veiga (2008). It uses the asymmetric linear losses incurred by VaR forecasts. The quantile loss (QL) is given by the following function:

$$
Q L_{t}^{a}=\left(\alpha-I_{t}(\alpha)\right)\left(y_{t}-\operatorname{VaR}_{t}^{a}\right)
$$

\subsection{Expected shortfall backtesting}

VaR is an elicitable risk measure, where a statistics $\phi(Y)$ of a random variable $Y$ is said to be elicitable if it minimises the expected value of a scoring function $S$ (Acerbi and Szekely (2014)):

$$
\phi=\arg \min _{x} E[S(x, Y)]
$$

It has been shown that VaR has several shortcomings, and that in particular is not able to capture tail risks beyond the $\alpha$-quantile (Artzner et al. (1999); Danielsson et al. (2001); Basel Committee (2013)). For these reasons, ES was introduced (Artzner et al. (1997, 1999)). To put it simply, ES is the expected loss given that the loss $L$ exceeds VaR $(E S=E[L \mid L>\operatorname{VaR}])$. Mathematically:

$$
E S_{\gamma}(X)=\frac{1}{\gamma} \int_{0}^{\gamma} \operatorname{VaR}_{\alpha}(X) d \alpha
$$

where $E S_{\gamma}(X)$ is ES of a random variable $X$ at level $\gamma$.

The transition from VaR to ES as the main market risk metric (Basel Committee, 2016) makes it necessary to have a reliable backtesting procedure of ES.

McNeil and Frey (2000) suggested an exceedance residual (ER) test that involves fitting a (volatility) model to the return data, estimating predictions of the mean and volatility, and then calculating the implied model residuals. Next, extreme value theory (EVT) can be applied, while treating the residuals as a white noise process, to model the tail of the marginal distribution. Lastly, the EVT model can be used to estimate the upper quantile of the marginal distribution. One can then test whether the expected value of ER is zero against the one-sided alternative that it is greater than zero or, equivalently, that ES is systematically underestimated.

The most recent test is the regression-based one of Bayer and Dimitriadis (2018), known as the ESR test. These authors use a joint regression framework for the quantile and the ES, and present two-sided and one-sided versions of the test. Suppose that $\hat{e_{t}}$ is the ES forecast and $Y_{t}$ is the $\log$ return. Then one can regress returns on the ES forecast as follows:

$$
Y_{t}=\alpha+\beta * \hat{e}_{t}+u_{t}^{e}
$$

where $E S_{\gamma}\left(u_{t}^{e} \mathscr{F}_{t-1}\right)=0$. One can then test the joint hypothesis that $\alpha$ is equal to 0 and $\beta$ is equal to $1, H_{0}:(\alpha, \beta)=(0,1)$, against the alternative that $H_{1}:(\alpha, \beta) \neq(0,1)$.

As the functional ES is not elicitable, these authors estimate semi-parametrically the following system: 


$$
\left\{\begin{array}{l}
Y_{t}=\alpha_{0}+\beta_{0} * \hat{e_{t}}+u_{t}^{q} \\
Y_{t}=\alpha_{1}+\beta_{1} * \hat{e_{t}}+u_{t}^{e}
\end{array}\right.
$$

where $\operatorname{VaR}_{\gamma}\left(u_{t}^{q} \mathscr{F}_{t-1}\right)=0$ and $E S_{\gamma}\left(u_{t}^{e} \mathscr{F}_{t-1}\right)=0$.

A Wald statistic is then carried out using the parameters $(\alpha, \beta)$ :

$$
T_{E S R}=\left((\alpha, \beta)^{\prime}-(0,1)^{\prime}\right)^{\prime} \Sigma_{E S}^{-1}\left((\alpha, \beta)^{\prime}-(0,1)^{\prime}\right)^{\prime},
$$

where $\hat{\Sigma_{E S}}$ is an estimator for the (asymptotic) covariance matrix of the M-estimator of the parameters $(\alpha, \beta)$. Hence, the test statistics follows asymptotically a $\chi_{2}^{2}$ distribution.

The test described above has been named the bivariate ESR test by Bayer and Dimitriadis (2018). Since in their opinion the possibility of underestimating risk is the main issue for regulators, they also suggest another regression-based backtesting procedure for the ES:

$$
Y_{t}-\hat{e_{t}}=\alpha+u_{t}^{e},
$$

where $E S_{\gamma}\left(u_{t}^{e} \mathscr{F}_{t-1}\right)=0$ and the null hypothesis is that $\alpha$ is zero. These are $t$-tests based on the asymptotic covariance.

\subsection{Model confidence set}

The backtesting procedures described above cannot help to select the best GARCH specification (even QL is not sufficiently informative). For this purpose we shall use instead the model confidence set (MCS) procedure introduced by Hansen et al. (2011). This consists of a sequence of tests that is used to construct a set of "superior" models (SSM) for which the null hypothesis of equal predictive ability (EPA) is not rejected.

Specifically, consider a set, $M^{0}$, that contains a finite number of models $\left(i=1, \ldots, m_{0}\right)$; these are evaluated in terms of a specified loss function for the $i$-th model at time $t, L_{i, t}$, where $t=1, \ldots, n$.

Let the performance variable be:

$$
d_{i j, t}=L_{i, t}-L_{j, t} \forall i, j \in M^{0}
$$

Then define the relative sample loss statistics

$$
\bar{d}_{i j}=\frac{1}{n} \sum_{t=1}^{n} d_{i j, t},
$$

and

$$
\bar{d}_{i .}=\frac{1}{m} \sum_{j \in M} \bar{d}_{i j} .
$$

Finally assume that

$$
\mu_{i j}=E\left(d_{i j}\right) \text { and } \mu_{i} .=E\left(d_{i} .\right)
$$

is finite and does not depend on $t \forall i, j \in M^{0}$.

Therefore, the set of superior models is defined as

$$
M^{*}=\left\{i \in M^{0}: \mu_{i j} \leq 0 \forall j \in M^{0}\right\} .
$$

In order to eliminate inferior elements of the set $M^{0}$ the following EPA hypotheses are tested

$$
\begin{gathered}
H_{0, M}: \mu_{i j}=0 \forall i, j \in M, \\
H_{A, M}: \mu_{i j} \neq 0 \text { for some } i, j \in M
\end{gathered}
$$

or

$$
\begin{gathered}
H_{0, M}: \mu_{i} .=0 \forall i, j \in M, \\
H_{A, M}: \mu_{i} . \neq 0 \text { for some } i, j \in M
\end{gathered}
$$

where $M \subset M^{0}$.

From these statistics the following $t$-statistics can be constructed

$$
t_{i j}=\frac{\bar{d}_{i j}}{\sqrt{\operatorname{vâ} r\left(\bar{d}_{i j}\right)}} \text { and } t_{i .}=\frac{\bar{d}_{i .}}{\sqrt{\operatorname{va} r\left(\bar{d}_{i .}\right)}} \text { for } i, j \in M \text {. }
$$

The two EPA hypotheses map into the two test statistics

$$
T_{R, M}=\max _{i, j \in M}\left|t_{i j}\right| \text { and } T_{\max , M}=\max _{i \in M} t_{i} .
$$

As mentioned before, the MCS procedure is a sequential testing procedure that removes the worst model at each step, until the 
hypothesis of EPA is not rejected for all models in the SSM. The choice of the worst model to be eliminated is made using an elimination rule that is coherent with the statistic tests defined above, namely:

$$
e_{\max , M}=\arg \max _{i \in M} \frac{\bar{d}_{i .}}{\operatorname{vâr}\left(\bar{d}_{i .}\right)}, e_{R, M}=\arg \max _{i}\left\{\sup _{j \in M} \frac{\bar{d}_{i j}}{\sqrt{\operatorname{vâ}\left(\bar{d}_{i j}\right)}}\right\} .
$$

To summarise, the MCS procedure consist of the following steps:

1 set $M=M^{0}$,

2 Test for the EPA hypothesis; if EPA is not rejected terminate the algorithm and set $M=M^{*}$, otherwise use the elimination rule and find the worst model,

\section{Remove the worst model, and go to step 2}

The MCS procedure requires a loss function. For the VaR the QL will be used. However, the choice of a loss function is not straightforward. Various papers have attempted to develop a consistent scoring function. Here we shall use the same loss function for ES backtesting as in Bayer and Dimitriadis (2018), which belongs to a class of functions originally introduced by Fissler and Ziegel (2016) in the context of forecast evaluation. They derived a consistent scoring function for $\left(\operatorname{VaR}_{\alpha}, E S_{\alpha}\right)$ in the following way.

Let $\alpha \in(0,1)$. Also let $\mathscr{F}$ be a class of distribution functions on $\mathscr{R}$ with finite first moments and unique $\alpha$-quantiles, and $A_{0}=\left\{x \in \mathscr{R}^{2}: x_{1} \geq x_{2}\right\}$. A scoring function $\mathrm{S}$ is then defined as follows: $A_{0} \times \mathscr{R} \rightarrow \mathscr{R}$ of the form

$$
S\left(x_{1}, x_{2}, y\right)=\left(1_{\left\{y \leq x_{1}\right\}}-\alpha\right) G_{1}\left(x_{1}\right)-1_{\left\{y \leq x_{1}\right\}} G_{1}(y)+G_{2}\left(x_{2}\right)\left(x_{2}-x_{1}+\frac{1}{\alpha} 1_{\left\{y \leq x_{1}\right\}}\left(x_{1}-y\right)\right)-\mathscr{G}_{2}\left(x_{2}\right)+a(y),
$$

where $G_{1}, G_{2}, \mathscr{G}_{2}, a: \mathscr{R} \rightarrow \mathscr{R}, \mathscr{G}_{2}^{\prime}=G_{2}, a$ is $\mathscr{F}$-integrable and $1_{\left(-\infty ; x_{1}\right]} G_{1}$ is $\mathscr{F}$-integrable $\forall x_{1} \in \mathscr{R}$, is $\mathscr{F}$-consistent for $\left(V a R_{\alpha}, E S_{\alpha}\right)$ if $G_{1}$ is increasing and $\mathscr{G}_{2}$ is increasing and convex. If $\mathscr{G}_{2}$ is strictly increasing and strictly convex, then $S$ is strictly $\mathscr{F}$-consistent for $\left(R_{\alpha}, E S_{\alpha}\right)$.

Bayer and Dimitriadis (2018) used the scoring function defined above as follows:

$$
S\left(V a R_{\alpha}^{t}, E S_{\alpha}^{t}, y_{t}\right)=\frac{1}{-E S_{\alpha}^{t}}\left(E S_{\alpha}^{t}-V a R_{\alpha}^{t}+\frac{\left(V a R_{\alpha}^{t}-y_{t}\right) 1_{\left\{y \leq x_{1}\right\}}}{\alpha}\right)+\log \left(-E S_{\alpha}^{t}\right),
$$

which is the 0-homogeneous loss function (see Dimitriadis and Bayer (2017); Taylor (2017); Patton et al. (2017), Barendse (2017) and Nolde and Ziegel (2017)).

\section{Empirical results}

As mentioned above, we estimate the following GARCH specifications: SGARCH, EGARCH, GJRGARCH and TGARCH. Normal (norm), skewed normal (snorm), Student's t (std), skewed Student's t (sstd), generalized error (ged) and skewed generalized error distributions (sged) are used. Finally, the mixture parameter is included; if this takes the value of one (TRUE) then the model becomes the distribution mixture GARCH, if it is instead set equal to zero (FALSE) it yields the MSGARCH specification. In total, 1176 GARCH models were estimated for each cryptocurrency following the maximum likelihood procedure described in detail in Aria et al. (2017).

The results were obtained from 1-step ahead VaR and ES predictions. A moving window with refitting at every step was used for the estimates and the predictions. The window size is $70 \%$ of the total number of observations. The models that did not fail the backtesting procedures were then used in the MCS procedure in order to obtain the best model or set of models. The p-values in the MCS procedure was set equal to $30 \%$.

\subsection{Value-at-risk results}

Table 2 reports p-values for the backtesting procedures and the MCS procedure for the superior set of models (SSM) for Bitcoin. As can be seen, 24 models out of 1176 satisfy the VaR and ES backtesting procedures and were selected by MCS as models with equal predictive power with respect to QL. Note that none of the single regime models was selected for the SSM.

Standard GARCH models prevail in the first and second regimes. Interestingly, the normal distribution prevails in the first regime, but whilst the Student's t distribution is appropriate for $70 \%$ of the models in the second regime. Mixture models represent approximately $60 \%$ of those selected for the SSM. It is also noteworthy that in the first regime a variety of specifications appear to be appropriate, whilst in the second standard GARCH and GJRGRACH account for more than $90 \%$ of the specifications. The same pattern emerges for the distribution functions: in the second regime the Student's t and normal distribution are chosen in $87.5 \%$ of the cases. Interestingly, no models with a skewed GED distribution are selected for the SSM, and none with skewed distributions in the second regime. Specifications that account for leverage effects at least in one of the regimes represent more than $75 \%$ of those selected for the SSM.

Table 3 shows the results for Ethereum. Only one model was selected. It is a mixture of GJRGARCH with Student's t distribution in the first regime and TGARCH with skewed Student's $t$ distribution in the second regime. 
Table 2

P-values of the Bitcoin backtesting and MCS procedures for the VaR loss function. The table reports the selected functional form for the first and second regime respectively, followed by the corresponding distributions and finally the mixture parameter: if this takes the value of one (TRUE) then the model becomes the distribution mixture GARCH, if it is instead set equal to zero (FALSE) it yields the MSGARCH specification.

\begin{tabular}{|c|c|c|c|c|c|c|c|}
\hline № & Model & UC & $\mathrm{CC}$ & DQ & ER & ESR & MCS \\
\hline 1 & tGARCH-sGARCH-sstd-std-TRUE & $63 \%$ & $84 \%$ & $99 \%$ & $68 \%$ & $87 \%$ & $100 \%$ \\
\hline 2 & tGARCH-gjrGARCH-sstd-std-TRUE & $61 \%$ & $83 \%$ & $100 \%$ & $81 \%$ & $80 \%$ & $100 \%$ \\
\hline 3 & eGARCH-sGARCH-snorm-std-TRUE & $86 \%$ & $89 \%$ & $99 \%$ & $71 \%$ & $97 \%$ & $100 \%$ \\
\hline 4 & sGARCH-sGARCH-norm-std-TRUE & $87 \%$ & $91 \%$ & $100 \%$ & $66 \%$ & $94 \%$ & $100 \%$ \\
\hline 5 & sGARCH-sGARCH-norm-std-FALSE & $85 \%$ & $91 \%$ & $100 \%$ & $58 \%$ & $96 \%$ & $100 \%$ \\
\hline 6 & sGARCH-gjrGARCH-norm-std-FALSE & $85 \%$ & $91 \%$ & $100 \%$ & $59 \%$ & $96 \%$ & $100 \%$ \\
\hline 7 & gjrGARCH-eGARCH-ged-std-TRUE & $59 \%$ & $81 \%$ & $100 \%$ & $66 \%$ & $71 \%$ & $100 \%$ \\
\hline 8 & gjrGARCH-gjrGARCH-norm-std-FALSE & $85 \%$ & $91 \%$ & $100 \%$ & $67 \%$ & $89 \%$ & $99 \%$ \\
\hline 9 & eGARCH-sGARCH-sstd-std-TRUE & $60 \%$ & $82 \%$ & $100 \%$ & $68 \%$ & $56 \%$ & $98 \%$ \\
\hline 10 & sGARCH-gjrGARCH-snorm-std-FALSE & $87 \%$ & $90 \%$ & $99 \%$ & $67 \%$ & $95 \%$ & $97 \%$ \\
\hline 11 & gjrGARCH-sGARCH-norm-ged-FALSE & $87 \%$ & $90 \%$ & $100 \%$ & $81 \%$ & $68 \%$ & $97 \%$ \\
\hline 12 & eGARCH-sGARCH-std-std-TRUE & $60 \%$ & $82 \%$ & $100 \%$ & $54 \%$ & $62 \%$ & $96 \%$ \\
\hline 13 & sGARCH-sGARCH-snorm-std-FALSE & $87 \%$ & $90 \%$ & $100 \%$ & $67 \%$ & $99 \%$ & $95 \%$ \\
\hline 14 & gjrGARCH-gjrGARCH-std-std-TRUE & $85 \%$ & $91 \%$ & $97 \%$ & $57 \%$ & $73 \%$ & $94 \%$ \\
\hline 15 & sGARCH-sGARCH-snorm-std-TRUE & $87 \%$ & $91 \%$ & $100 \%$ & $65 \%$ & $94 \%$ & $94 \%$ \\
\hline 16 & eGARCH-gjrGARCH-norm-norm-TRUE & $87 \%$ & $90 \%$ & $99 \%$ & $84 \%$ & $85 \%$ & $94 \%$ \\
\hline 17 & sGARCH-gjrGARCH-snorm-norm-TRUE & $87 \%$ & $90 \%$ & $99 \%$ & $72 \%$ & $91 \%$ & $78 \%$ \\
\hline 18 & sGARCH-tGARCH-std-std-TRUE & $89 \%$ & $92 \%$ & $100 \%$ & $77 \%$ & $81 \%$ & $74 \%$ \\
\hline 19 & sGARCH-sGARCH-norm-ged-FALSE & $59 \%$ & $81 \%$ & $100 \%$ & $65 \%$ & $81 \%$ & $74 \%$ \\
\hline 20 & tGARCH-sGARCH-norm-ged-TRUE & $59 \%$ & $81 \%$ & $100 \%$ & $54 \%$ & $91 \%$ & $71 \%$ \\
\hline 21 & tGARCH-sGARCH-std-norm-FALSE & $59 \%$ & $81 \%$ & $100 \%$ & $72 \%$ & $65 \%$ & $67 \%$ \\
\hline 22 & gjrGARCH-sGARCH-norm-std-FALSE & $85 \%$ & $91 \%$ & $100 \%$ & $59 \%$ & $97 \%$ & $67 \%$ \\
\hline 23 & sGARCH-sGARCH-std-norm-FALSE & $59 \%$ & $81 \%$ & $100 \%$ & $58 \%$ & $84 \%$ & $66 \%$ \\
\hline 24 & gjrGARCH-sGARCH-std-std-TRUE & $60 \%$ & $82 \%$ & $92 \%$ & $59 \%$ & $92 \%$ & $66 \%$ \\
\hline
\end{tabular}

\section{Table 3}

P-values of the Ethereum backtesting and MCS procedures for the VaR loss function. The table reports the selected functional form for the first and second regime respectively, followed by the corresponding distributions and finally the mixture parameter: if this takes the value of one (TRUE) then the model becomes the distribution mixture GARCH, if it is instead set equal to zero (FALSE) it yields the MSGARCH specification.

\begin{tabular}{llllllll}
\hline № & Model & UC & CC & DQ & ER & ESR & MCS \\
\hline 1 & gjrGARCH-tGARCH-std-sstd-TRUE & $96 \%$ & $97 \%$ & $99 \%$ & & $100 \%$ & $65 \%$ \\
\hline
\end{tabular}

Table 4 reports the results for Ripple. Only eight models satisfied all backtesting procedures and were selected by the MCS procedure. For the first regime models with standard GARCH or TGARCH were chosen by this procedure. For the second regime $50 \%$ of the models selected for the SSM have a TGARCH specification and 25\% of them has either a standard GARCH or GJRGARCH specification. In the first regime normal and skewed normal distributions prevail, while in the second the Student's $t$ and skewed Student's t distributions are found to be appropriate for all models. The same percentage of mixture models and Markov-switching models are selected for the SSM. Specifications accounting for leverage effects at least in one of the regimes represent more than $75 \%$ of those in the SSM.

Table 5 shows that MCS selects the highest percentage of models satisfying the backtesting procedures in the case of Litecoin; in total, 33 models out of 1176 were chosen. In the first regime TGARCH models represent $40 \%$ of those selected, standard GARCH ones $30 \%$ and EGARCH and GJRGARCH the rest; the most common distribution is the normal, followed by the skewed normal and Student's t. In the second regime standard GARCH models exceed 50\%, and the Student's t and skewed Student's t distributions are

\section{Table 4}

P-values of the Ripple backtesting and MCS procedures for the VaR loss function. The table reports the selected functional form for the first and second regime respectively, followed by the corresponding distributions and finally the mixture parameter: if this takes the value of one (TRUE) then the model becomes the distribution mixture GARCH, if it is instead set equal to zero (FALSE) it yields the MSGARCH specification.

\begin{tabular}{|c|c|c|c|c|c|c|c|}
\hline № & Model & UC & CC & DQ & ER & ESR & MCS \\
\hline 1 & sGARCH-sGARCH-snorm-sstd-TRUE & $30 \%$ & $57 \%$ & $100 \%$ & $54 \%$ & $66 \%$ & $100 \%$ \\
\hline 2 & tGARCH-gjrGARCH-sstd-sstd-FALSE & $11 \%$ & $28 \%$ & $87 \%$ & $89 \%$ & $61 \%$ & $100 \%$ \\
\hline 3 & sGARCH-tGARCH-sged-sstd-FALSE & $11 \%$ & $28 \%$ & $96 \%$ & $100 \%$ & $84 \%$ & $100 \%$ \\
\hline 4 & sGARCH-tGARCH-snorm-std-TRUE & $12 \%$ & $29 \%$ & $95 \%$ & $6 \%$ & $58 \%$ & $100 \%$ \\
\hline 5 & tGARCH-gjrGARCH-norm-std-FALSE & $30 \%$ & $57 \%$ & $96 \%$ & $99 \%$ & $10 \%$ & $99 \%$ \\
\hline 6 & tGARCH-tGARCH-norm-sstd-FALSE & $44 \%$ & $68 \%$ & $94 \%$ & $100 \%$ & $10 \%$ & $98 \%$ \\
\hline 7 & sGARCH-tGARCH-norm-std-TRUE & $11 \%$ & $28 \%$ & $84 \%$ & $100 \%$ & $99 \%$ & $69 \%$ \\
\hline 8 & sGARCH-sGARCH-snorm-std-TRUE & $94 \%$ & $95 \%$ & $100 \%$ & $79 \%$ & $85 \%$ & $64 \%$ \\
\hline
\end{tabular}


Table 5

P-values of the Litecoin backtesting and MCS procedures for the VaR loss function. The table reports the selected functional form for the first and second regime respectively, followed by the corresponding distributions and finally the mixture parameter: if this takes the value of one (TRUE) then the model becomes the distribution mixture GARCH, if it is instead set equal to zero (FALSE) it yields the MSGARCH specification.

\begin{tabular}{|c|c|c|c|c|c|c|c|}
\hline № & Model & UC & $\mathrm{CC}$ & DQ & ER & ESR & MCS \\
\hline 1 & gjrGARCH-tGARCH-norm-sstd-TRUE & $51 \%$ & $78 \%$ & $62 \%$ & $100 \%$ & $85 \%$ & $100 \%$ \\
\hline 2 & tGARCH-sGARCH-snorm-sstd-TRUE & $52 \%$ & $79 \%$ & $100 \%$ & $56 \%$ & $51 \%$ & $100 \%$ \\
\hline 3 & sGARCH-sGARCH-norm-sstd-FALSE & $83 \%$ & $91 \%$ & $97 \%$ & $60 \%$ & $83 \%$ & $100 \%$ \\
\hline 4 & sGARCH-sGARCH-norm-std-FALSE & $83 \%$ & $91 \%$ & $93 \%$ & $70 \%$ & $82 \%$ & $100 \%$ \\
\hline 5 & gjrGARCH-gjrGARCH-norm-sged-TRUE & $50 \%$ & $78 \%$ & $99 \%$ & $53 \%$ & $59 \%$ & $100 \%$ \\
\hline 6 & gjrGARCH-tGARCH-sstd-std-TRUE & $51 \%$ & $78 \%$ & $100 \%$ & $63 \%$ & $67 \%$ & $100 \%$ \\
\hline 7 & tGARCH-sGARCH-sged-sstd-TRUE & $51 \%$ & $78 \%$ & $100 \%$ & $50 \%$ & $71 \%$ & $100 \%$ \\
\hline 8 & sGARCH-sGARCH-std-sstd-TRUE & $83 \%$ & $93 \%$ & $100 \%$ & $58 \%$ & $90 \%$ & $99 \%$ \\
\hline 9 & tGARCH-gjrGARCH-std-sstd-TRUE & $84 \%$ & $94 \%$ & $100 \%$ & $57 \%$ & $51 \%$ & $99 \%$ \\
\hline 10 & sGARCH-sGARCH-norm-sged-TRUE & $83 \%$ & $91 \%$ & $100 \%$ & $54 \%$ & $76 \%$ & $98 \%$ \\
\hline 11 & sGARCH-sGARCH-snorm-std-TRUE & $53 \%$ & $75 \%$ & $99 \%$ & $63 \%$ & $99 \%$ & $98 \%$ \\
\hline 12 & sGARCH-sGARCH-sstd-std-TRUE & $83 \%$ & $93 \%$ & $100 \%$ & $66 \%$ & $80 \%$ & $98 \%$ \\
\hline 13 & gjrGARCH-sGARCH-sstd-std-TRUE & $83 \%$ & $93 \%$ & $100 \%$ & $68 \%$ & $54 \%$ & $97 \%$ \\
\hline 14 & tGARCH-tGARCH-snorm-ged-TRUE & $53 \%$ & $80 \%$ & $100 \%$ & $86 \%$ & $79 \%$ & $97 \%$ \\
\hline 15 & sGARCH-sGARCH-std-std-TRUE & $51 \%$ & $78 \%$ & $100 \%$ & $57 \%$ & $74 \%$ & $97 \%$ \\
\hline 16 & tGARCH-tGARCH-norm-ged-TRUE & $50 \%$ & $78 \%$ & $100 \%$ & $58 \%$ & $65 \%$ & $95 \%$ \\
\hline 17 & tGARCH-gjrGARCH-ged-sstd-TRUE & $86 \%$ & $94 \%$ & $100 \%$ & $86 \%$ & $65 \%$ & $93 \%$ \\
\hline 18 & tGARCH-sGARCH-ged-sstd-TRUE & $51 \%$ & $78 \%$ & $100 \%$ & $66 \%$ & $79 \%$ & $93 \%$ \\
\hline 19 & gjrGARCH-gjrGARCH-std-std-TRUE & $50 \%$ & $78 \%$ & $100 \%$ & $52 \%$ & $54 \%$ & $93 \%$ \\
\hline 20 & tGARCH-sGARCH-sstd-std-TRUE & $84 \%$ & $94 \%$ & $100 \%$ & $88 \%$ & $56 \%$ & $84 \%$ \\
\hline 21 & gjrGARCH-sGARCH-std-sged-TRUE & $50 \%$ & $78 \%$ & $99 \%$ & $54 \%$ & $66 \%$ & $81 \%$ \\
\hline 22 & eGARCH-sGARCH-ged-sged-TRUE & $50 \%$ & $78 \%$ & $100 \%$ & $54 \%$ & $67 \%$ & $78 \%$ \\
\hline 23 & gjrGARCH-eGARCH-norm-std-FALSE & $50 \%$ & $78 \%$ & $100 \%$ & $57 \%$ & $53 \%$ & $76 \%$ \\
\hline 24 & eGARCH-tGARCH-snorm-ged-TRUE & $50 \%$ & $78 \%$ & $100 \%$ & $57 \%$ & $60 \%$ & $74 \%$ \\
\hline 25 & sGARCH-tGARCH-snorm-ged-TRUE & $50 \%$ & $78 \%$ & $100 \%$ & $52 \%$ & $54 \%$ & $72 \%$ \\
\hline 26 & eGARCH-gjrGARCH-snorm-sged-TRUE & $50 \%$ & $78 \%$ & $100 \%$ & $52 \%$ & $53 \%$ & $69 \%$ \\
\hline 27 & tGARCH-sGARCH-sged-std-TRUE & $51 \%$ & $78 \%$ & $99 \%$ & $56 \%$ & $57 \%$ & $68 \%$ \\
\hline 28 & sGARCH-sGARCH-snorm-sged-TRUE & $83 \%$ & $93 \%$ & $100 \%$ & $51 \%$ & $85 \%$ & $64 \%$ \\
\hline 29 & tGARCH-sGARCH-sstd-sstd-TRUE & $82 \%$ & $91 \%$ & $100 \%$ & $86 \%$ & $51 \%$ & $63 \%$ \\
\hline 30 & tGARCH-eGARCH-sstd-snorm-TRUE & $85 \%$ & $95 \%$ & $100 \%$ & $69 \%$ & $53 \%$ & $53 \%$ \\
\hline 31 & tGARCH-sGARCH-norm-ged-FALSE & $51 \%$ & $78 \%$ & $99 \%$ & $99 \%$ & $79 \%$ & $52 \%$ \\
\hline 32 & sGARCH-eGARCH-std-sged-FALSE & $83 \%$ & $91 \%$ & $99 \%$ & $64 \%$ & $81 \%$ & $38 \%$ \\
\hline 33 & tGARCH-sGARCH-std-ged-FALSE & $51 \%$ & $78 \%$ & $100 \%$ & $94 \%$ & $82 \%$ & $34 \%$ \\
\hline
\end{tabular}

also selected in more than $50 \%$ of the cases for the SSM. Mixture models represent more than $80 \%$ of those in the SSM. Specifications allowing for leverage at least in one regime account for approximately $76 \%$ of those in the SSM.

\subsection{Expected shortfall results}

Table 6 reports p-values for all backtesting procedures and the MCS procedure to choose the best set of models for Bitcoin. As can be seen 25 models out of 1176 satisfy the VaR and ES backtesting procedures and were selected by MCS as models with equal predictive power with respect to the joint loss function. Note that none of the single regime models was selected for the SSM.

The results from the MCS procedure with a joint loss function are mostly the same as those for QL, since SSM is constructed from the same set of models that did not fail the VaR and ES backtesting procedures. Standard GARCH models prevail in the first and second regimes. Interestingly, the normal distribution prevails in the first regime, but in the second the Student's $t$ distribution is selected in $70 \%$ of the cases. Mixture models represent $60 \%$ of those in the SSM. In the first regime there is more variety of specifications, whilst in the second standard GARCH and GJRGRACH represent $90 \%$ of the chosen models. Similarly, the Student's t and normal distribution prevail in the second regime being selected in $88 \%$ of the cases. No models with a skewed GED distribution are included in the SSM, and none with a skewed distribution in the second regime. Specifications accounting for leverage effects at least in one of the regimes represent $80 \%$ of those in the SSM.

Table 7 shows the results for Ethereum, which are essentially the same as for QL.

Table 8 reports the results for Ripple. Only five models did not fail the backtesting procedures and were selected by the MCS procedure. In the first regime standard GARCH or TGARCH models were chosen by MCS procedure; in the second a TGARCH specification is selected for the SSM in $60 \%$ of the cases, and GARCH or GJRGARCH ones in $20 \%$ of them. Normal and skewed normal distributions prevail in the first regime, whilst Student's $t$ and skewed Student's t distributions are chosen in all cases in the second. Markov-switching and mixture models represent respectively $60 \%$ and $40 \%$ of those included in the SSM. Specifications with leverage effects at least in one of the regimes represent $80 \%$ of the total in the SSM.

Table 9 shows the results of the MCS procedure for Litecoin with a joint loss function. In total 47 models were chosen, the highest percentage of models satisfying the VaR and ES backtesting procedures. Standard GARCH is chosen in one third of the cases in the first regime, and in approximately half of them in the second. The Student's t distribution and its skewed version prevail in both 
Table 6

P-values of the Bitcoin backtesting and MCS procedures for the joint loss function. The table reports the selected functional form for the first and second regime respectively, followed by the corresponding distributions and finally the mixture parameter: if this takes the value of one (TRUE) then the model becomes the distribution mixture GARCH, if it is instead set equal to zero (FALSE) it yields the MSGARCH specification.

\begin{tabular}{|c|c|c|c|c|c|c|c|}
\hline № & Model & UC & CC & DQ & ER & ESR & MCS \\
\hline 1 & eGARCH-sGARCH-snorm-std-TRUE & $86 \%$ & $89 \%$ & $99 \%$ & $71 \%$ & $97 \%$ & $100 \%$ \\
\hline 2 & eGARCH-sGARCH-sstd-std-TRUE & $60 \%$ & $82 \%$ & $100 \%$ & $68 \%$ & $56 \%$ & $100 \%$ \\
\hline 3 & gjrGARCH-gjrGARCH-std-std-TRUE & $85 \%$ & $91 \%$ & $97 \%$ & $57 \%$ & $73 \%$ & $100 \%$ \\
\hline 4 & tGARCH-sGARCH-sstd-std-TRUE & $63 \%$ & $84 \%$ & $99 \%$ & $68 \%$ & $87 \%$ & $100 \%$ \\
\hline 5 & sGARCH-sGARCH-norm-std-FALSE & $85 \%$ & $91 \%$ & $100 \%$ & $58 \%$ & $96 \%$ & $100 \%$ \\
\hline 6 & sGARCH-sGARCH-norm-std-TRUE & $87 \%$ & $91 \%$ & $100 \%$ & $66 \%$ & $94 \%$ & $100 \%$ \\
\hline 7 & gjrGARCH-sGARCH-norm-ged-FALSE & $87 \%$ & $90 \%$ & $100 \%$ & $81 \%$ & $68 \%$ & $100 \%$ \\
\hline 8 & gjrGARCH-gjrGARCH-norm-std-FALSE & $85 \%$ & $91 \%$ & $100 \%$ & $67 \%$ & $89 \%$ & $100 \%$ \\
\hline 9 & tGARCH-gjrGARCH-sstd-std-TRUE & $61 \%$ & $83 \%$ & $100 \%$ & $81 \%$ & $80 \%$ & $100 \%$ \\
\hline 10 & sGARCH-gjrGARCH-norm-std-FALSE & $85 \%$ & $91 \%$ & $100 \%$ & $59 \%$ & $96 \%$ & $100 \%$ \\
\hline 11 & eGARCH-sGARCH-std-std-TRUE & $60 \%$ & $82 \%$ & $100 \%$ & $54 \%$ & $62 \%$ & $100 \%$ \\
\hline 12 & sGARCH-sGARCH-norm-ged-FALSE & $59 \%$ & $81 \%$ & $100 \%$ & $65 \%$ & $81 \%$ & $100 \%$ \\
\hline 13 & sGARCH-sGARCH-std-norm-FALSE & $59 \%$ & $81 \%$ & $100 \%$ & $58 \%$ & $84 \%$ & $100 \%$ \\
\hline 14 & gjrGARCH-eGARCH-ged-std-TRUE & $59 \%$ & $81 \%$ & $100 \%$ & $66 \%$ & $71 \%$ & $99 \%$ \\
\hline 15 & tGARCH-sGARCH-std-norm-FALSE & $59 \%$ & $81 \%$ & $100 \%$ & $72 \%$ & $65 \%$ & $99 \%$ \\
\hline 16 & tGARCH-sGARCH-norm-ged-TRUE & $59 \%$ & $81 \%$ & $100 \%$ & $54 \%$ & $91 \%$ & $99 \%$ \\
\hline 17 & sGARCH-gjrGARCH-snorm-std-FALSE & $87 \%$ & $90 \%$ & $99 \%$ & $67 \%$ & $95 \%$ & $99 \%$ \\
\hline 18 & gjrGARCH-sGARCH-std-std-TRUE & $60 \%$ & $82 \%$ & $92 \%$ & $59 \%$ & $92 \%$ & $99 \%$ \\
\hline 19 & sGARCH-sGARCH-snorm-std-FALSE & $87 \%$ & $90 \%$ & $100 \%$ & $67 \%$ & $99 \%$ & $97 \%$ \\
\hline 20 & sGARCH-tGARCH-std-std-TRUE & $89 \%$ & $92 \%$ & $100 \%$ & $77 \%$ & $81 \%$ & $97 \%$ \\
\hline 21 & eGARCH-gjrGARCH-norm-norm-TRUE & $87 \%$ & $90 \%$ & $99 \%$ & $84 \%$ & $85 \%$ & $93 \%$ \\
\hline 22 & sGARCH-gjrGARCH-snorm-norm-TRUE & $87 \%$ & $90 \%$ & $99 \%$ & $72 \%$ & $91 \%$ & $79 \%$ \\
\hline 23 & tGARCH-gjrGARCH-norm-std-TRUE & $86 \%$ & $91 \%$ & $100 \%$ & $67 \%$ & $89 \%$ & $48 \%$ \\
\hline 24 & sGARCH-gjrGARCH-norm-norm-TRUE & $87 \%$ & $90 \%$ & $99 \%$ & $61 \%$ & $88 \%$ & $43 \%$ \\
\hline 25 & gjrGARCH-sGARCH-norm-std-FALSE & $85 \%$ & $91 \%$ & $100 \%$ & $59 \%$ & $97 \%$ & $39 \%$ \\
\hline
\end{tabular}

Table 7

P-values of the Ethereum backtesting and MCS procedures for the joint loss function. The table reports the selected functional form for the first and second regime respectively, followed by the corresponding distributions and finally the mixture parameter: if this takes the value of one (TRUE) then the model becomes the distribution mixture GARCH, if it is instead set equal to zero (FALSE) it yields the MSGARCH specification.

\begin{tabular}{llllllll}
\hline № & Model & UC & CC & DQ & ER & ESR & MCS \\
\hline 1 & gjrGARCH-tGARCH-std-sstd-TRUE & $96 \%$ & $97 \%$ & $99 \%$ & $100 \%$ & $65 \%$ & $100 \%$ \\
\hline
\end{tabular}

\section{Table 8}

P-values of the Ripple backtesting procedure and MCS procedures for the joint loss function. The table reports the selected functional form for the first and second regime respectively, followed by the corresponding distributions and finally the mixture parameter: if this takes the value of one (TRUE) then the model becomes the distribution mixture GARCH, if it is instead set equal to zero (FALSE) it yields the MSGARCH specification.

\begin{tabular}{|c|c|c|c|c|c|c|c|}
\hline № & Model & UC & CC & DQ & ER & ESR & MCS \\
\hline 1 & sGARCH-sGARCH-snorm-sstd-TRUE & $30 \%$ & $57 \%$ & $100 \%$ & $54 \%$ & $66 \%$ & $100 \%$ \\
\hline 2 & sGARCH-tGARCH-sged-sstd-FALSE & $11 \%$ & $28 \%$ & $96 \%$ & $100 \%$ & $84 \%$ & $100 \%$ \\
\hline 3 & sGARCH-tGARCH-snorm-std-TRUE & $12 \%$ & $29 \%$ & $95 \%$ & $6 \%$ & $58 \%$ & $91 \%$ \\
\hline 4 & tGARCH-tGARCH-norm-sstd-FALSE & $44 \%$ & $68 \%$ & $94 \%$ & $100 \%$ & $10 \%$ & $79 \%$ \\
\hline 5 & tGARCH-gjrGARCH-sstd-sstd-FALSE & $11 \%$ & $28 \%$ & $87 \%$ & $89 \%$ & $61 \%$ & $45 \%$ \\
\hline
\end{tabular}

regime, whilst the percentages for the normal distribution and its skewed version are $40 \%$ and $4 \%$ respectively in the two regimes. The corresponding percentages for the GED distribution are $44 \%$ and $18 \%$ respectively. Mixture models are selected in $80 \%$ of the cases for the SSM. Specifications with leverage effects are also chosen in $80 \%$ of the cases at least in one regime.

\section{Conclusions}

This paper has used VaR and ES backtesting as well as the MCS procedure to select the best model or superior set of GARCH volatility models for four of the main cryptocurrencies, namely Bitcoin, Ethereum, Ripple and Litecoin. Two-regime GARCH models are found to produce better VaR and ES predictions than single-regime models.

Surprisingly, both the QL and joint loss function results suggest a standard GARCH model for Bitcoin. As for Ethereum, the MCS procedure selects a regime mixture model with a GJR GARCH specification and Student's t distribution in the first regime and a TGARCH model with a skewed Student's t distribution in the second regime. Concerning Ripple, for the first regime the QL results 
Table 9

P-values of the Litecoin backtesting and MCS procedures for the joint loss function. The table reports the selected functional form for the first and second regime respectively, followed by the corresponding distributions and finally the mixture parameter: if this takes the value of one (TRUE) then the model becomes the distribution mixture GARCH, if it is instead set equal to zero (FALSE) it yields the MSGARCH specification.

\begin{tabular}{|c|c|c|c|c|c|c|c|}
\hline № & Model & $\mathrm{UC}$ & $\mathrm{CC}$ & DQ & ER & ESR & MCS \\
\hline 1 & sGARCH-sGARCH-norm-std-FALSE & $83 \%$ & $91 \%$ & $93 \%$ & $70 \%$ & $82 \%$ & $100 \%$ \\
\hline 2 & sGARCH-sGARCH-norm-sstd-FALSE & $83 \%$ & $91 \%$ & $97 \%$ & $60 \%$ & $83 \%$ & $100 \%$ \\
\hline 3 & gjrGARCH-tGARCH-norm-sstd-TRUE & $51 \%$ & $78 \%$ & $62 \%$ & $100 \%$ & $85 \%$ & $100 \%$ \\
\hline 4 & tGARCH-sGARCH-snorm-sstd-TRUE & $52 \%$ & $79 \%$ & $100 \%$ & $56 \%$ & $51 \%$ & $100 \%$ \\
\hline 5 & tGARCH-sGARCH-sged-sstd-TRUE & $51 \%$ & $78 \%$ & $100 \%$ & $50 \%$ & $71 \%$ & $100 \%$ \\
\hline 6 & gjrGARCH-gjrGARCH-norm-sged-TRUE & $50 \%$ & $78 \%$ & $99 \%$ & $53 \%$ & $59 \%$ & $100 \%$ \\
\hline 7 & sGARCH-sGARCH-std-sstd-TRUE & $83 \%$ & $93 \%$ & $100 \%$ & $58 \%$ & $90 \%$ & $100 \%$ \\
\hline 8 & sGARCH-sGARCH-snorm-std-TRUE & $53 \%$ & $75 \%$ & $99 \%$ & $63 \%$ & $99 \%$ & $100 \%$ \\
\hline 9 & tGARCH-sGARCH-ged-sstd-TRUE & $51 \%$ & $78 \%$ & $100 \%$ & $66 \%$ & $79 \%$ & $99 \%$ \\
\hline 10 & sGARCH-sGARCH-sstd-std-TRUE & $83 \%$ & $93 \%$ & $100 \%$ & $66 \%$ & $80 \%$ & $97 \%$ \\
\hline 11 & gjrGARCH-sGARCH-std-sged-TRUE & $50 \%$ & $78 \%$ & $99 \%$ & $54 \%$ & $66 \%$ & $97 \%$ \\
\hline 12 & tGARCH-gjrGARCH-std-sstd-TRUE & $84 \%$ & $94 \%$ & $100 \%$ & $57 \%$ & $51 \%$ & $96 \%$ \\
\hline 13 & sGARCH-sGARCH-norm-sged-TRUE & $83 \%$ & $91 \%$ & $100 \%$ & $54 \%$ & $76 \%$ & $95 \%$ \\
\hline 14 & sGARCH-sGARCH-std-std-TRUE & $51 \%$ & $78 \%$ & $100 \%$ & $57 \%$ & $74 \%$ & $95 \%$ \\
\hline 15 & gjrGARCH-tGARCH-sstd-std-TRUE & $51 \%$ & $78 \%$ & $100 \%$ & $63 \%$ & $67 \%$ & $94 \%$ \\
\hline 16 & tGARCH-tGARCH-norm-ged-TRUE & $50 \%$ & $78 \%$ & $100 \%$ & $58 \%$ & $65 \%$ & $94 \%$ \\
\hline 17 & gjrGARCH-sGARCH-sstd-std-TRUE & $83 \%$ & $93 \%$ & $100 \%$ & $68 \%$ & $54 \%$ & $92 \%$ \\
\hline 18 & tGARCH-sGARCH-sstd-sstd-TRUE & $82 \%$ & $91 \%$ & $100 \%$ & $86 \%$ & $51 \%$ & $91 \%$ \\
\hline 19 & eGARCH-tGARCH-snorm-ged-TRUE & $50 \%$ & $78 \%$ & $100 \%$ & $57 \%$ & $60 \%$ & $89 \%$ \\
\hline 20 & tGARCH-sGARCH-std-ged-FALSE & $51 \%$ & $78 \%$ & $100 \%$ & $94 \%$ & $82 \%$ & $88 \%$ \\
\hline 21 & eGARCH-sGARCH-snorm-sged-TRUE & $50 \%$ & $78 \%$ & $100 \%$ & $50 \%$ & $53 \%$ & $88 \%$ \\
\hline 22 & sGARCH-eGARCH-std-sged-FALSE & $83 \%$ & $91 \%$ & $99 \%$ & $64 \%$ & $81 \%$ & $87 \%$ \\
\hline 23 & tGARCH-tGARCH-snorm-ged-TRUE & $53 \%$ & $80 \%$ & $100 \%$ & $86 \%$ & $79 \%$ & $86 \%$ \\
\hline 24 & tGARCH-gjrGARCH-ged-sstd-TRUE & $86 \%$ & $94 \%$ & $100 \%$ & $86 \%$ & $65 \%$ & $85 \%$ \\
\hline 25 & gjrGARCH-eGARCH-norm-std-FALSE & $50 \%$ & $78 \%$ & $100 \%$ & $57 \%$ & $53 \%$ & $85 \%$ \\
\hline 26 & tGARCH-sGARCH-sstd-std-TRUE & $84 \%$ & $94 \%$ & $100 \%$ & $88 \%$ & $56 \%$ & $85 \%$ \\
\hline 27 & sGARCH-sGARCH-sged-sstd-FALSE & $50 \%$ & $78 \%$ & $100 \%$ & $55 \%$ & $60 \%$ & $82 \%$ \\
\hline 28 & gjrGARCH-eGARCH-sstd-ged-TRUE & $50 \%$ & $78 \%$ & $99 \%$ & $53 \%$ & $67 \%$ & $79 \%$ \\
\hline 29 & eGARCH-gjrGARCH-snorm-sged-TRUE & $50 \%$ & $78 \%$ & $100 \%$ & $52 \%$ & $53 \%$ & $79 \%$ \\
\hline 30 & sGARCH-tGARCH-snorm-ged-TRUE & $50 \%$ & $78 \%$ & $100 \%$ & $52 \%$ & $54 \%$ & $71 \%$ \\
\hline 31 & eGARCH-sGARCH-ged-sged-TRUE & $50 \%$ & $78 \%$ & $100 \%$ & $54 \%$ & $67 \%$ & $70 \%$ \\
\hline 32 & sGARCH-tGARCH-norm-ged-TRUE & $50 \%$ & $78 \%$ & $100 \%$ & $52 \%$ & $60 \%$ & $69 \%$ \\
\hline 33 & gjrGARCH-gjrGARCH-std-std-TRUE & $50 \%$ & $78 \%$ & $100 \%$ & $52 \%$ & $54 \%$ & $68 \%$ \\
\hline 34 & sGARCH-eGARCH-std-snorm-TRUE & $50 \%$ & $78 \%$ & $97 \%$ & $51 \%$ & $59 \%$ & $64 \%$ \\
\hline 35 & sGARCH-eGARCH-ged-sged-TRUE & $50 \%$ & $78 \%$ & $100 \%$ & $53 \%$ & $63 \%$ & $63 \%$ \\
\hline 36 & sGARCH-tGARCH-sged-sstd-FALSE & $50 \%$ & $78 \%$ & $100 \%$ & $63 \%$ & $68 \%$ & $61 \%$ \\
\hline 37 & eGARCH-sGARCH-snorm-sged-FALSE & $50 \%$ & $78 \%$ & $100 \%$ & $52 \%$ & $58 \%$ & $59 \%$ \\
\hline 38 & tGARCH-eGARCH-sstd-snorm-TRUE & $85 \%$ & $95 \%$ & $100 \%$ & $69 \%$ & $53 \%$ & $59 \%$ \\
\hline 39 & tGARCH-sGARCH-norm-ged-FALSE & $51 \%$ & $78 \%$ & $99 \%$ & $99 \%$ & $79 \%$ & $56 \%$ \\
\hline 40 & gjrGARCH-eGARCH-std-std-TRUE & $50 \%$ & $78 \%$ & $96 \%$ & $61 \%$ & $72 \%$ & $55 \%$ \\
\hline 41 & eGARCH-tGARCH-sstd-sstd-TRUE & $53 \%$ & $80 \%$ & $100 \%$ & $71 \%$ & $69 \%$ & $52 \%$ \\
\hline 42 & sGARCH-sGARCH-snorm-sged-TRUE & $83 \%$ & $93 \%$ & $100 \%$ & $51 \%$ & $85 \%$ & $50 \%$ \\
\hline 43 & tGARCH-sGARCH-sged-std-TRUE & $51 \%$ & $78 \%$ & $99 \%$ & $56 \%$ & $57 \%$ & $43 \%$ \\
\hline 44 & sGARCH-eGARCH-std-sged-TRUE & $50 \%$ & $78 \%$ & $98 \%$ & $55 \%$ & $68 \%$ & $42 \%$ \\
\hline 45 & sGARCH-gjrGARCH-sstd-ged-TRUE & $50 \%$ & $78 \%$ & $100 \%$ & $58 \%$ & $71 \%$ & $40 \%$ \\
\hline 46 & gjrGARCH-tGARCH-snorm-ged-FALSE & $51 \%$ & $78 \%$ & $99 \%$ & $96 \%$ & $64 \%$ & $38 \%$ \\
\hline 47 & eGARCH-sGARCH-std-std-TRUE & $50 \%$ & $78 \%$ & $100 \%$ & $62 \%$ & $59 \%$ & $35 \%$ \\
\hline
\end{tabular}

lead to the choice of standard GARCH and TGARCH models, and the joint loss function ones suggest a standard GARCH specification; for the second regime TGARCH models account for most of the SSM. Lastly, for Litecoin standard GARCH models prevail in both regimes.

It is noteworthy that specifications allowing for leverage at least in one regime account for more than 75\% of the SSM for all four cryptocurrencies. In particular, symmetric GARCH models are the most appropriate for one regime in the case of Bitcoin, Ethereum and Litecoin, but for the other in most cases an asymmetric functional form is found to be preferable; this confirms the importance of using a Markov-switching framework - Dyhrberg (2016), for instance, had not found leverage effects in his study that does not allow for different regimes. Mixture GARCH models prevail for almost all cryptocurrencies with both a QL function and a joint loss function.

On the whole, our findings imply that cryptocurrencies exhibit high volatility and leverage effects (at least in one regime). Tworegime models yield better results in terms of prediction for both VaR and ES. Mixture distribution models outperform Markovswitching ones. Our analysis also indicates that using single-regime GARCH models may yield incorrect VaR and ES predictions, and hence result in ineffective risk-management, portfolio optimisation, pricing of derivative securities etc. These could be improved by using instead the model specifications suggested by our analysis, from which both investors and regulators (such as the US Securities and Exchange Commission (SEC), which is planning to regulate the cryptocurrency exchanges) can benefit. 
Future work will use intraday data to address the issue of the large number of the observations required by some of the tests carried out in the present paper, and also estimate multivariate GARCH models, e.g. to examine the linkages between Altcoins and Bitcoin.

\section{References}

Acerbi, C., Szekely, B., 2014. Backtesting expected shortfall. Risk Mag. 27, 76-81.

Alexander, C., Lazar, E., 2006. Normal mixture GARCH (1, 1): applications to exchange rate modelling. J. Appl. Econom. 21 (3), $307-336$.

Alizadeh, A.H., Nomikos, N.K., Pouliasis, P.K., 2008. A Markov regime switching approach for hedging energy commodities. J. Bank. Financ. 32, 1970-1983.

Ardia, D., Bluteau, K., Boudt, K., Catania, L., 2018a. Forecasting risk with Markov-switching GARCH models: a large-scale performance study. Int. J. Forecast. 34, $733-747$.

Ardia, D., Bluteau, K., Rüede, M., 2018b. Regime Changes in Bitcoin GARCH Volatility Dynamics. Finance Research Letters. https://doi.org/10.1016/j.frl.2018.08. 009.

Artzner, P.F., 1997. Thinking coherently. Risk 10 (11), 68-71.

Artzner, P., Delbaen, F., Eber, J.M., Heath, D., 1999. Coherent measures of risk. Math. Financ. 9 (3), $203-228$.

Barendse, S., 2017. Interquantile Expectation Regression. Available at. Tinbergen Institute Discussion Paper 2017-034/III. https://ssrn.com/abstract=2937665.

Basel Committee, 2013. Fundamental Review of the Trading Book: a Revised Market Risk Framework. Technical Report. Available at. Bank for International Settlements. http://www.bis.org/publ/bcbs265.pdf.

Basel Committee, 2016. Minimum Capital Requirements for Market Risk. Technical Report. Available at. Bank for International Settlements. http://www.bis.org/ bcbs/publ/d352.pdf.

Bauwens, L., Preminger, A., Rombouts, J.V.K., 2010. Theory and inference for a Markov switching GARCH model. Econ. J. 13, 218-244. https://doi.org/10.1111/j. 1368-423X.2009.00307.x.

Bauwens, L., Backer, B.D., Dufays, A., 2014. A Bayesian method of change-point estimation with recurrent regimes: application to GARCH models. J. Empir. Finance 29, 207-229. https://doi.org/10.1016/j.jempfin.2014.06.008.

Bayer, S., Dimitriadis, T., 2018. Regression based expected shortfall backtesting. arXiv preprint arXiv:1801.04112.

Bohl, M.T., Salm, C.A., Wilfling, B., 2011. Do individual index futures investors destabilize the underlying spot market? J. Futures Mark. 31, 81-101.

Bollerslev, T., 1986. Generalized autoregressive conditional heteroskedasticity. J. Econom. 31, 307-327. https://doi.org/10.1016/0304-4076(86)90063-1.

Bollerslev, T., 1987. A conditionally heteroskedastic time series model for speculative prices and rates of return. Rev. Econ. Stat. 69 , 542-547. https://doi.org/10. 2307/1925546.

Bollerslev, T., Chou, R.Y., Kroner, K., 1992. ARCH modeling in finance: a review of the theory and empirical evidence. J. Econ. 52, 5-59. https://doi.org/10.1016/ 0304-4076(92)90064-X.

Bollerslev, T., Engle, R.F., Nelson, D.B., 1994. Handbook of Econometrics. North-Holland. chapter 49.. pp. $2959-3038$.

Bouoiyour, J., Selmi, R., 2016. Bitcoin: a beginning of a new phase? Econ. Bull. 36 (3), 1430-1440. http://www.accessecon.com/Pubs/EB/2016/Volume36/EB-16V36-I3-P142.pdf.

Cai, J., 1994. A Markov model of switching-regime ARCH. J. Bus. Econ. Stat. 12 (3), 309-316.

Caporale, G.M., Pittis, N., Spagnolo, N., 2003. IGARCH models and structural breaks. Appl. Econ. Lett. 10, $765-768$.

Christoffersen, P.F., 1998. Evaluating interval forecasts. Int. Econ. Rev. 39, 841-862.

Chu, J., Chan, S., Nadarajah, S., Osterrieder, J., 2017. GARCH modelling of cryptocurrencies. J. Risk Financ. Manag. 10 (4), 17.

Cifter, A., Ozun, A., 2007. The predictive performance of asymmetric normal mixture GARCH in risk management: evidence from Turkey. MPRA Paper 2489.

Danielsson, J., Embrechts, P., Goodhart, C., Keating, C., Muennich, F., Renault, O., Shin, H.S., 2001. An Academic Response to Basel II. Financial Markets Group Special Papers. available at. https://EconPapers.repec.org/RePEc:fmg:fmgsps:sp130.

Dimitriadis, T., Bayer, S., 2017. A Joint Quantile and Expected Shortfall Regression Framework. arXiv:1704.02213 [math.ST]. .

Dueker, M.J., 1997. Markov switching in GARCH processes and mean reverting stock-market volatility. J. Bus. Econ. Stat. 15 (1), $26-34$.

Dyhrberg, A.H., 2016. Bitcoin, gold and the dollar - A GARCH volatility analysis. Financ. Res. Lett. 16, 85-92. https://doi-org.lcproxy.shu.ac.uk/10.1016/j.frl.2015. 10.008.

Engle, R.F., 1982. Autoregressive conditional heteroscedasticity with estimates of the variance of United Kingdom inflation. Econometrica 50, 987-1008. https://doi. org $/ 10.2307 / 1912773$

Engle, R.F., 2004. Risk and volatility: econometric models and financial practice. Am. Econ. Rev. 94, 405-420. https://doi.org/10.1257/0002828041464597.

Fantazzini, D., Nigmatullin, E., Sukhanovskaya, V., Ivliev, S., 2016. Everything You Always Wanted to Know About Bitcoin Modelling but Were Afraid to Ask. Available at SSRN:. https://ssrn.com/abstract $=2794622$.

Fissler, T., Ziegel, J.F., 2016. Higher order elicitability and Osband's principle. Ann. Stat. 44 (4), 1680-1707.

Glaser, F., Haferhorn, M., Weber, M.C., Zimmarmann, K., Siering, Mb, 2014. Bitcoin - Asset or Currency? Revealing Users' Hidden Intentions. ECIS 2014 Tel Aviv.

Glosten, L.R., Jagannathan, R., Runkle, D.E., 1993. On the relation between the expected value and the volatility of the nominal excess return on stocks. J. Financ. 48 , 1779-1801. https://doi.org/10.1111/j.1540-6261.1993.tb05128.x.

González-Rivera, G., Lee, T.H., Mishra, S., 2004. Forecasting volatility: a reality check based on option pricing, utility function, Value-at-Risk, and predictive likelihood. Int. J. Forecast. 20, 629-645.

Gray, S.F., 1996. Modeling the conditional distribution of interest rates as a regime-switching process. J. Financ. econ. 42 (1), $27-62$.

Gronwald, M., 2014. The Economics of Bitcoins - Market Characteristics and Price Jumps (No. 5121). CESifo Working Paper, (5121). Available at SSRN:. https://ssrn. com/abstract $=2548999$.

Haas, M., Mittnik, S., Paolella, M.S., 2004. A new approach to Markov-switching GARCH models. J. Financ. Econom. 2, 493-530. https://doi.org/10.1093/jjfinec/ nbh020.

Hamilton, J.D., Susmel, R., 1994. Autoregressive conditional heteroskedasticity and changes in regime. J. Econ. 64 (1-2), $307-333$.

Hansen, P.R., Lunde, A., 2005. A forecast comparison of volatility models: does anything beat a GARCH (1,1)? J. Appl. Econ. 20 (7), 873-889.

Hansen, P.R., Lunde, A., Nason, J.M., 2011. The model confidence set. Econometrica 79 (2), $453-497$.

Henry, O.T., 2009. Regime switching in the relationship between equity returns and short-term interest rates in the UK. J. Bank. Financ. 33 , $405-414$.

Katsiampa, P., 2017. Volatility estimation for Bitcoin: a comparison of GARCH models. Econ. Lett. 158, 3-6.

Klaassen, F., 2002. Improving GARCH volatility forecasts with regimeswi;Tching GARCH. Advances in Markov-switching Models. Springer-Verlag, pp. 223-254.

Kupiec, P.H., 1995. Techniques for verifying the accuracy of risk measurement models. J. Deriv. 3.

Manganelli, S., Engle, R.F., 2004. CAViaR: conditional autoregressive Value at Risk by regression quantiles. J. Bus. Econ. Stat. 22 , $367-381$.

McAleer, M., Da Veiga, B., 2008. Single-index and portfolio models for forecasting Value-at-Risk thresholds. J. Forecast. 27, $217-235$.

McNeil, A.J., Frey, R., 2000. Estimation of tail-related risk measures for heteroscedastic financial time series: an extreme value approach. J. Empir. Finance 7 (3-4), 271-300.

Nakamoto, S., 2009. Bitcoin: A Peer-to-Peer Electronic Cash System. https://Bitcoin.org/Bitcoin.pdf.

Nelson, D.B., 1991. Conditional heteroskedasticity in asset returns: a new approach. Econometrica 59, 347-370. https://doi.org/10.2307/2938260.

Nolde, N., Ziegel, J.F., 2017. Elicitability and backtesting: perspectives for banking regulation. Ann. Appl. Stat. 11 (4), $1833-1874$.

Patton, A.J., Ziegel, J.F., Chen, R., 2017. Dynamic Semiparametric Models for Expected Shortfall (and Value-at-Risk). arXiv:1707.05108 [q-fin.EC]. .

Taylor, J.W., 2017. Forecasting value at risk and expected shortfall using a semiparametric approach based on the asymmetric Laplace distribution. J. Bus. Econ. Stat. 0 (0), 1-13.

Wilfling, B., 2009. Volatility regime-switching in European exchange rates prior to monetary unification. J. Int. Money Finance 28, 240-270.

Zakoian, J.M., 1994. Threshold heteroskedastic models. J. Econ. Dyn. Control 18, 931-955. https://doi.org/10.1016/0165-1889(94)90039-6. 